\title{
L'autotraduire littéraire : un espace pour (re)penser le sujet traduisant et la poétique du traduire.
}

Nadine Celotti

\section{(2) OpenEdition}

\section{Journals}

Édition électronique

URL : http://journals.openedition.org/rief/1598

DOI : $10.4000 /$ rief. 1598

ISSN : 2240-7456

Éditeur

Seminario di filologia francese

Référence électronique

Nadine Celotti, «L'autotraduire littéraire : un espace pour (re)penser le sujet traduisant et la poétique du traduire. », Revue italienne d'études françaises [En ligne], 7 | 2017, mis en ligne le 15 novembre 2017, consulté le 24 avril 2019. URL : http://journals.openedition.org/rief/1598 ; DOI : 10.4000/rief.1598

Ce document a été généré automatiquement le 24 avril 2019.

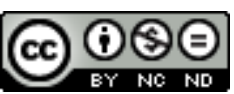

Les contenus de la RIEF sont mis à disposition selon les termes de la Licence Creative Commons Attribution - Pas d'Utilisation Commerciale - Pas de Modification 4.0 International. 


\title{
L'autotraduire littéraire : un espace pour (re)penser le sujet traduisant et la poétique du traduire.
}

\author{
Nadine Celotti
}

1 Habiter deux langues - ou davantage -, penser (entre) deux langues, vivre (dans) deux langues et écrire (dans) deux langues offrent au sujet un espace potentiel pour s'autotraduire. Tout au long de l'histoire du traduire littéraire, du Moyen Âge et de la Renaissance $^{1}$ - Louis Des Masures, pour ne pas toujours citer que Du Bellay, avec ses CEuvres poétiques et Carmina ${ }^{2}$ - jusqu'à aujourd'hui, avec des œuvres de l'exilé forcé ou volontaire, de l'émigré, du dominé, du privilégié, divers chemins balisés par les contextes culturels-politiques et par les expériences personnelles ont conduit à l'activité de l'autotraduire.

2 Les autotraducteurs expérimentent deux langues d'écriture d'un même texte avec des vécus différents par rapport à leur langue maternelle ou d'origine - bilingues dès l'enfance, tardifs, de survie, de culture, contraints - et avec des réalités diverses par rapport à la langue de leur noyau familial et à celle de leur communauté, à la langue locale et à celle du maître. Mus, entre autres, par une conscience d'affirmation identitaire, par des pulsions ou par un sentiment de contrainte, comme un geste politique ou un acte de révolte, en quête, parfois, de légitimité éditoriale sur un marché plus vaste.

3 Certains ont écrit dans leur langue d'origine et se sont traduits dans leur langue d'adoption comme Panaiit Istrati, du roumain au français. D'autres ont quitté leur langue d'origine et l'ont retrouvée par la traduction comme Marco Micone, du français à l'italien. Et d'autres encore ont parcouru les deux directions comme Jean-Joseph Rabearivelo, «le prince des poètes malgaches ", du malgache vers le français et inversement, pour ne pas toujours citer que Samuel Beckett ou Nancy Huston dans l'espace de la langue française. Des autotraducteurs sédentaires, qui vivent au quotidien leur dualité de langues au sein de leur communauté, et des autotraducteurs migrateurs ${ }^{3}$, qui ont adopté une langue autre que la première dans leur mouvement de vie. 
4 Le phénomène de l'autotraduire se décline en une multiplicité de langues (et dialectes) aux statuts et prestiges différents, langues conquérantes et langues soumises, langues centrales et langues périphériques, qui embrassent nombre de pays allant au-delà du traditionnel Occident ${ }^{4}$.

5 Cette pluralité et diversité de situations cohabitent dans un ensemble nommé "autotraduction", devenu un domaine d'exploration scientifique à part entière, si ce n'est «un territoire pionnier, prometteur, arpenté par des cohortes de jeunes chercheurs $»^{5}$. À partir de Fitch ${ }^{6}$, «qui fait figure de pionnier $»^{7}$, les premières études se sont focalisées sur des autotraducteurs de renom comme Samuel Beckett - qui ne manque jamais à l'appel $»^{8}$, même s'« il ne semble guère représentatif $»^{9}-$, Julian/Julien Green ou Nancy Huston, pour ensuite élargir leur terrain d'exploration et s'intéresser plus

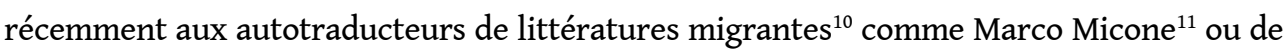
langues périphériques ${ }^{12}$ comme l'auteur et autotraducteur breton Pierre-Jakez Hélias ${ }^{13}$, pour rester dans l'espace de la langue française. Au tournant du $\mathrm{XX}^{\mathrm{e}}$ siècle, d'innombrables colloques - où l'Italie (Pescara en 2010, Udine en 2010 et 2012, Bologne en 2011) a joué un rôle majeur - , des numéros spéciaux de revues ${ }^{14}$ et de nombreuses monographies ${ }^{15} s^{\prime}$ ouvrent à des sujets de recherche qui vont au-delà des études de cas et montrent combien "l'auto-traduction constitue un espace propre ${ }^{16}$. De ce fait, des distinctions se sont dessinées.

Des autotraductions entre langues lointaines, comme les langues occidentales et les langues orientales, et celles entre langues proches, comme les langues latines ${ }^{17}$. L'autotraduction horizontale et l'autotraduction verticale selon la symétrie ou l'asymétrie des deux langues dans leur rapport de pouvoir ${ }^{18}$. L'autotraduction naturalisante, décentrée, $(\mathrm{re})$ créatrice $^{19}$ selon l'approche traductive choisie par l'écrivain. L'autotraduction différée, simultanée ou consécutive ${ }^{20}$ selon l'intervalle temporel qui s'écoule entre les deux textes. L'autotraduction transparente et la traduction opaque ${ }^{21}$ selon la présence ou l'absence d'éléments paratextuels dévoilant ou cachant l'existence du texte original.

7 Une importante variété de disciplines et d'approches s'entrecroisent: « Il s'agit en effet d'un champ d'étude [l'autotraduction] interdisciplinaire par excellence $»^{22}$. Littérature générale et comparée, littérature postcoloniale, littérature migrante, sociologie littéraire, sociolinguistique, génétique textuelle et Translations Studies - discipline universitaire (re)connue à l'échelle internationale, nommée le plus souvent «traductologie » dans l'espace francophone ${ }^{23}$, mais qui voile les différences épistémologiques et les débats sur sa dénomination. "Ainsi la poétique de la traduction débordera toujours ce qui se présente comme traductologie $»^{24}$. Sans oublier que "Traduction, translation et Übersetzung ne "se traduisent" pas mutuellement sauf dans les dictionnaires $»^{25}$. Les Translation Studies ont reconnu l'existence de l'autotraduction en tant qu'objet d'étude à part entière dès la parution du premier dictionnaire de la discipline ${ }^{26}$ en lui donnant son statut d'entrée et elles l'ont confirmé ensuite dans leurs différents ouvrages lexicographiques de référence ${ }^{27}$. Si, avant les années 2000, elles dénonçaient la marginalité des recherches, elles constatent aujourd'hui le foisonnement des études sur la "self-translation ». L'ouvrage The Bilingual Text: History and Theory of Literary Self-Translation de Hockenson et Munson ${ }^{28}$, devenu la référence bibliographique traductologique de base, est salué comme «la tentative la plus ambitieuse de tracer la terra incognita de la "self-translation" $"{ }^{29}$.

8 Certes, la langue anglaise joue le rôle de lingua franca dans les Translation Studies ${ }^{30}$, mais il faut remarquer que l'ensemble des études sur l'autotraduction ne reste pas sous 
l'hégémonie de la langue anglaise. D'autres langues comme l'espagnol - l'Espagne représente non seulement "aujourd'hui un des principaux foyers de l'activité autotraductionnelle $»^{31}$, mais aussi les études de l'espace hispanophone connaissent un réel dynamisme ${ }^{32}-$, l'italien - les recherches italiennes ${ }^{33}$ sont stimulées entre autres par la présence des dialectes et par la littérature des migrants ${ }^{34}$ - et le français - initialement par le biais des recherches au Canada puis de celles de la France qui se développeront une quinzaine d'années plus tard ${ }^{35}$. Ces quatre langues, voire plus avec le catalan ${ }^{36}$, dialoguent et dynamisent ce lieu d'échanges de différentes langues-cultures, ce qui ne peut qu'enrichir la pensée et le regard traductologique.

9 La distinction qu'il est d'usage d'établir pour explorer l'autotraduction - l'acte de traduire et le résultat de cette opération ${ }^{37}$ - porte à focaliser l'activité dans ce qu'elle a de spécifique et pousse à substantiver le verbe " autotraduire " pour la nommer. À la suite de Meschonnic «[j]e dis poétique du traduire, plutôt que "poétique de la traduction", pour marquer qu'il s'agit de l'activité, à travers ses produits $»^{38}$. C'est une activité générée par un sujet autotraduisant bien visible contrairement à un traducteur allographe qui n'accède que difficilement à la visibilité. Visibilité et invisibilité, deux mots fondamentaux qui habitent, voire hantent, les Translations Studies et qui pourraient être repensés grâce aux études sur l'autotraduire.

Le traducteur auctorial est bien visible hors texte : aussi bien dans les péritextes, devenus objets de recherches et qui alimentent les réflexions sur le sujet ${ }^{39}$, que dans les épitextes comme les entretiens avec l'autotraducteur ${ }^{40}$. Voilà une invitation à poursuivre les toutes premières études ${ }^{41}$ sur « l'étayage de la traduction elle-même » ${ }^{42}$. De plus, le traducteur auctorial est naturellement nommé sans complexe traductologique - ce qui pourrait redonner vie aux réflexions de Berman pour une critique des traductions: "Ainsi la question qui est le traducteur? doit-elle être fermement posée face à une traduction ${ }^{43}$.

Le traducteur auctorial, reconnu comme étant le protagoniste de l'écriture d'un texte dans une langue autre, est également bien visible dans le texte. Il crée, expérimente, adapte, réécrit. Il est légitimé à réécrire en tant que traducteur privilégié ${ }^{44}$, puisqu'il est également le créateur du texte à traduire. Il réécrit mais il traduit. "L'auteur ne réinvente pas une nouvelle œuvre littéraire, l'original précède la réécriture $»^{45}$. Tandis que le traducteur allographe est tout au plus qualifié de fidèle, loyal, sourcier ou cibliste, comme si son écriture n'était pas prise en compte. « Revaloriser la traduction implique qu'elle soit une écriture. Sans quoi, c'est une importune ${ }^{46}$.

12 L'écriture, la grande oubliée de la critique de la traduction - trop souvent enlisée dans la dualité du signe, la forme et le sens. Or, «[1]e texte et la traduction ne sont pas la même chose, on le sait. Mais la seconde relève du même processus d'écriture et les points communs entre ces deux formes d'écriture sont plus nombreux que leurs différences $»^{47}$.

Lire l'écriture du texte traduit conduit à comprendre si le sujet traduisant s'est inscrit prosodiquement et rythmiquement dans le texte, s'il est allé à la recherche de la poétique du traduire.

C'est justement ce regard sur l'écriture de la part des études de l'autotraduire qui, à mes yeux, est à saisir pour faire avancer les réflexions sur le traduire dans son ensemble. Henri Meschonnic l'avait déjà vu : «C'est le discours, et l'écriture, qu'il faut traduire. La banalité même $~^{48}$. Le texte traduit comme le texte autotraduit est un texte écrit - ou désécriit ${ }^{49}$, quand le sujet traduisant n'a pas été à l'écoute du rythme de l'écriture. 

texte traduit en mouvement ${ }^{51}$ - parallèlement à celles de l'autotraduction collaborative ${ }^{52}$ - qui explorent l'intervention de l'auteur à différents degrés sur la traduction de ses textes - tracent des orientations intéressantes pour (re)penser le travail du sujet traduisant.

L'étude de Weismann ${ }^{53}$ sur la genèse de poèmes de Paul Celan traduits en français me paraît éclairante. Celan n'est pas un autotraducteur entier : «Je ne sais pas me traduire, moi-même en français $"^{54}$ dit-il, mais c'est un autotraducteur collaboratif. Il est intervenu sur la traduction de ses poèmes en donnant «des consignes de correction très précises [...] en l'accordant à [sa] poétique $»^{55}$. Dans Spät und tief, par exemple, Celan modifie la traduction du titre de son poème proposée par Denise Naville ${ }^{56}$ «Tardif et profond» en "Tard, profondément». Il transforme la coordination entre les deux adjectifs en allemand rendue en français littéralement par la traductrice. On entend sa poétique. «"Lire", c'est "entendre" » nous dit Émile Benveniste ${ }^{57}$.

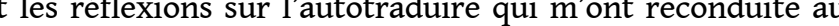
loisir de lire Benveniste, notamment ses notes sur le langage poétique de Baudelaire ${ }^{58}$, "ou plus précisément du "discours poétique" "59. Pour mieux saisir ce que "fait» le discours poétique pour être entendu et réécrit par le sujet traduisant.

Le principe premier me semble être que, en $/{ }^{60}$ poésie, les mots ne sont pas des signes, au / sens saussurien. Dès qu'on fait de la poésie, on quitte la convention des signes, qui régit / le langage ordinaire. ${ }^{61}$

C'est une des conditions essentielles du / jeu poétique : que le mot ne signifie pas (seulement), mais qu'il / évoque. ${ }^{62}$

18 Le dépassement du signe tel que Benveniste le met au jour, bien souligné par la rature de "seulement ${ }^{63}$ entre parenthèses, nous ramène à la question de la dualité du signe, dualité qui va au détriment de la prosodie et du rythme dans l'activité du sujet traduisant, comme l'a bien montré Meschonnic. "C'est alors une écriture, l'organisation d'une subjectivation dans le discours telle qu'elle transforme les valeurs de la langue en valeurs de discours. On ne peut plus continuer à les penser dans les termes coutumiers du signe » ${ }^{64}$.

Dans Sémiologie du langage, Benveniste soutient: «[e]n conclusion, il faut dépasser la notion saussurienne du signe comme principe unique, dont dépendraient à la fois la structure et le fonctionnement de la langue $"^{65}$. Dans les notes sur Baudelaire, il semble y avoir un aboutissement de ce dépassement dans le discours poétique : le mot «évoque ", il ne signifie pas. Cette pensée ouvre résolument de nouvelles voies à parcourir pour la poétique du traduire et invite à renouveler la lecture de Meschonnic.

20 À mes yeux, les travaux de l'autotraduire, notamment ceux de la genèse des textes autotraduits, permettent d'enrichir une pensée du traduire tendant vers l'écoute du sujet autotraduisant et du sujet traduisant, au-delà du signe.

«En effet, l'autotraduction [...] pose à la traduction comme à l'approche génétique des questions passionnantes $»^{66}$. 


\section{NOTES}

1. Cf. J. C. Santoyo Julio César, "Autotraducciones : una perspectiva historico », dans Meta, 50-3, 2007, p. 858-867 ; J. C. Santoyo, «Esbozo de una historia de la autotraducción », dans C. Lagarde et H. Tanquiero (dir.), L'autotraduction aux frontières de la langue et de la culture, Limoges, LambertLucas, 2013, p. 23-35 ; C. Galderisi et J.-J. Vincensini (dir.), La traduction entre Moyen Âge et Renaissance. Médiations, auto-traductions et traductions secondes, Turnhout, Brepols Publishers, 2017.

2. Dans lesquelles on peut lire : «Epitre premièrement faite latine : et depuis rendue française, par le même auteur ", N. Cernogora, " Poésie ", dans V. Duché (dir.), Histoire des traductions en langue française $\mathrm{XV}^{e}$ et XVI $\mathrm{X}^{e}$ siècles, Paris, Verdier, 2015, p. 1098.

3. Cf. R. Grutman, «Francophonie et autotraduction », dans Interfrancophonies, 6, 2015, p. 1-17.

4. Cf. P. Puccini, "Avant-propos. Pour une cartographie de l'autotraduction", dans Interfrancophonies, 6, 2015, p. I-XII.

5. C. Lagarde, «Avant-propos. L'autotraduction, terra incognita?», dans C. Lagarde et H. Tanquiero (dir.), op. cit., p. 12.

6. B. T. Fitch, «The Status of Self-Translation", dans Texte. Revue de critique et de théorie littéraire, 4, 1986, p. 111-125.

7. C. Lagarde, op. cit., p. 9.

8. R. Grutman, «L'autotraduction, de la galerie de portraits à la galaxie des langues ", dans A. Ferraro et R. Grutman (dir.), L'autotraduction littéraire, Paris, Classiques Garnier, 2016, p. 40.

9. R. Grutman, « Autotraduction, asymétrie, extraterritorialité », dans C. Lagarde et H. Tanquiero (dir.), op. cit., p. 42.

10. A. Ferraro (dir.), Oltreoceano, «L'autotraduzione nelle letterature migranti », 5, 2011.

11. Étudié notamment par P. Puccini. Cf., entre autres, P. Puccini, «L'autotraduction comme performance : Non era per noi di Marco Micone », dans C. Lagarde et H. Tanqueiro (dir.), op.cit., p. 133-144.

12. Cf. Pour la langue bretonne, par exemple, cf. E. Hupel, « Le cœur et l'esprit : déchirements et stratégies d'autotraduction chez quelques auteurs bretons ", dans Glottopol, 25, 2015, p. 124-135.

13. D. ar Rouz, «De l'autotraduction à la traduction de soi : éléments de réflexion bretonne ", dans Glottopol, 25, 2015, p. 103-123.

14. Des revues de littérature, de traduction ou de sociolinguistique de différents pays en différentes langues. Par exemple, Atelier de traduction, 7, 2007 ; Quaderns. Revista de traducció, 16, 2009 ; Oltreoceano, 5, 2011; Orbis Litterarum, 68/3, 2013; Tradução em revista, 16, 2014 ; Interfrancophonies, 6, 2015 ; Glottopol, 25, 2015 ; Ticontre. Teoria Testo Traduzione, 7, 2017.

15. Pour une bibliographie complète et mise à jour, cf. E. Dantes, Bibliography self translation http://www.academia.edu/30722381/Bibliography_self_translation_27-01.01.17. Cf. aussi A. Ferraro et R. Grutman, « Bibliographie », dans A. Ferraro et R. Grutman (dir.), op. cit., p. 221- 246.

16. M. Oustinoff, Bilinguisme d'écriture et auto-traduction, Julien Green, Samuel Beckett, Vladimir Nabokov, Paris, L'Harmattan, 2001, p. 12.

17. H. Tanqueiro, cité par Autotrad (équipe de recherche sur l'autotraduction), "L'autotraduction littéraire comme domaine de recherche », dans Atelier de traduction, 7, 2007, p. 94.

18. R. Grutman, "Autotraduction, asymétrie, extraterritorialité ", dans C. Lagarde et H. Tanquiero (dir.), op. cit., 2013, p. 37-44. Pour l'asymétrie, cf. aussi C. Lagarde, «Des langues minorées aux "langues mineures": autotraduction littéraire et sociolinguistique, une confrontation productive », dans Glottopol, 25, 2015, p. 2-13. 
19. M. Oustinoff, op. cit.

20. R. Grutman, "Manuscrits, traduction et autotraduction», dans C. Montini (dir.), Traduire. Genèse du choix, Paris, Edition des archives contemporaines, 2016, p. 120.

21. X. M. Dasilva, «L'opacité de l'autotraduction », dans A. Ferraro et R. Grutman (dir.), op. cit., 2016, p. 103-118.

22. Autotrad, op. cit., p. 91.

23. M. Oustinoff, «Les "Translations Studies" et le tournant traductologique ", dans J. Nowitcki, M. Oustinoff (dir.), Traduction et Mondialisation, Hermès, 49, 2007, p. 21-28.

24. H. Meschonnic, Poétique du traduire, Paris, Verdier, 1999, p. 125.

25. A. Berman, Jacques Amyot, traducteur français, Paris, Belin, 2012, p. 89.

26. "Auto-translation (or Self-translation)", dans M. Shuttleworth and M. Cowie, Dictionary of Translation Studies, Manchester, St. Jerome Publishing, 1997, p. 13.

27. R. Grutman, "Auto-translation », dans M. Baker, (eds.), Encyclopedia of Translation Studies, London/New York, Routledge, $1^{\text {ère }}$ éd., 1998, p. 17-20; R. Grutman, "Self-translation ", dans M. Baker and G. Saldanha (eds.), Encyclopedia of Translation Studies, London/New York, Routledge, 2 ème éd., 2009, p. 257-260; C. Montini, "Self-translation ", dans Y. Gambier and L. van Doorslaer (eds.), Handbook of Translation Studies, Amsterdam/Philadelphia, J. Benjamins, vol. 1, 2010, p. 306-308.

28. J. W. Hockenson et M. Munson, The Bilingual Text: History and Theory of Literary Self-Translation, Manchester, St Jerome Press, 2007.

29. Cf. R. Grutman, « Self-translation », cit., p. 257.

30. R. Grutman, « Manuscrits, traduction et autotraduction », cit., p. 115.

31. A. Ferraro et R. Grutman, « Avant-propos. L'autotraduction littéraire : cadres contextuels et dynamiques textuelles ", dans A. Ferraro et R. Grutman (dir.), op. cit., p. 9.

32. Cf. X. M. Dasilva, Estudios sobre la autotraducción en el espacio ibérico, Peter Lang, Bern 2013 ; J.-C. Santoyo, "Consideraciones acerca del estatus actual de la autotraducción en la península ibérica ", dans Glottopol, 25, 2015, p. 48-58.

33. Par exemple, A. Ceccherelli et al. (dir.), Autotraduzione e riscrittura, Bologna, Bononia University, Press, 2013.

34. Cf. A. Ferraro (dir.), « L'autotraduzione nelle letterature migranti », Oltreoceano, 5, 2011.

35. C. Lagarde, op. cit., p. 19.

36. Cf. C. Lagarde et H. Tanquiero (dir.), op. cit.

37. A. Ferraro et R. Grutman, op. cit., p. 7.

38. H. Meschonnic, Poétique du traduire, cit. p. 11.

39. Cf. X. M. Dasilva, «La autotraducción transparente y la autotraducción opaca », dans X. M. Dasilva et $\mathrm{H}$. Tanqueiro (dir.), Aproximaciones a la autotraducción, Vigo, Editorial Academia del Hispanismo, 2011, p.45-68, et notamment pour le «le pacte autotraductif» cf. A. Ferraro, "Traduit par l'auteur. Sur le pacte autotraductif», dans A. Ferraro et R. Grutman, op. cit., p. 121-140.

40. Par exemple, l'entretien avec Felicia Mihali par Elena-Brânduşa Steiciuc, dans Atelier de la traduction, 7, 2007, p. 15-23.

41. Cf. D. Risterucci-Roudnicky, Introduction à l'analyse des œuvres traduites, Paris, Colin, 2008 ; M. Nowotna et A. Moghani (dir.), Les traces du traducteur, Paris, Inalco Cedrom, 2010 ; E. Chiara, Traduzione e paratesto, Bologna, Bonomia University Press, 2012.

42. Expression utilisée par A. Berman pour dire tous les paratextes de traduction dans Pour une critique des traductions : John Donne, cit., p. 68.

43. A. Berman, Pour une critique des traductions: John Donne, cit., p. 73.

44. Cf. H. Tanqueiro, "Un traductor privilegiado: el autotraductor», Quaderns. Revista de Traducció, 3, 1999, p. 19-27.

45. Autotrad, op. cit., p. 94. 
46. H. Meschonnic, Poétique du traduire, cit., p. 28.

47. C. Montini, «Introduction », dans C. Montini (dir.), op. cit., 2016, p. ii.

48. H. Meschonnic, Poétique du traduire, cit., p. 12.

49. Cf. H. Meschonnic, "Traduire : écrire ou désécrire ", dans S. Hibbs et M. Martinez (dir.), Traduction adaptation réécriture dans le monde hispanique contemporain, Toulouse, Presses Universitaires du Mirail, 2006, p. 21.

50. Cf. par exemple : P. Sardin-Damestoy, Samuel Beckett auto-traducteur ou l'art de «l'empêchement ", Artois, Artois Presses Université, 2002 ; D. Weismann, « Entre contrôle et confiance : Paul Celan correcteur de ses traductions françaises ", dans C. Montini (dir.), op. cit., p. 129-144 ; R. Grutman, «Manuscrits, traduction et autotraduction», dans C. Montini (dir.), op.cit., p.115-128; C. Montini, "Genèse des textes et autotraduction. Le texte dans tous ses états ", dans A. Ferraro et R. Grutman (dir.), op. cit., p. 169-188.

51. La génétique de la traduction s'affirme aujourd'hui comme une "discipline émergente ». Cf. les numéros spéciaux de revue comme Genesis, F. Durand-Bogaert (dir.), « Traduction », 38, 2014 ; Transalpina, V. Agostini-Ouafi et A. Lavieri (dir.), «Poétiques des archives: Genèse des traductions et communautés de pratique », 18, 2015 ; Linguistica Antverpiensia, A. Cordingley et C. Montini (dir.), « Genetic translation studies : an emerging discipline », 14, 2015.

52. Cf. V. Sperti, «La traduction littéraire collaborative entre privilège auctorial et contrôle traductif », dans A. Ferraro, et R. Grutman (dir.), op. cit., p. 141-167.

53. D. Weismann, op. cit.

54. Propos de Celan dans une lettre. Cité par D. Weismann, op. cit., p. 136.

55. D. Weismann, op. cit., p. 135.

56. La traduction publiée est signée par D. Neville et elle cache de ce fait l'autotraduction collaborative, cependant l'intervention de l'auteur est bien dévoilée grâce au travail sur la genèse du texte de D. Weismann (Ibid.). Cf. également R. Grutman, «Manuscrits, traduction et autotraduction ", cit., p. 125.

57. É. Benveniste, Dernières leçons. Collège de France 1968 et 1969, Paris, EHESS, Gallimard, Seuil, 2012, p. 133.

58. É. Benveniste, Baudelaire, Limoges, Lambert-Lucas, 2011. Manuscrits conservés à la Bibliothèque de France, les seuls feuillets datés sont de 1967 (p. 8.). Les notes de Benveniste, présentées et transcrites par Chloé Laplantine en 2011, font actuellement l'objet de lectures plurielles et sont reconnues comme étant riches en perspectives innovantes. Cf. la revue Semen, «Les notes manuscrites de Benveniste sur la langue de Baudelaire », 33, 2012, en ligne, https:// semen.revues.org/9442.

59. I. Fenoglio, «Benveniste auteur d'une recherche inachevée sur "le discours poétique" et non d'un "Baudelaire" ", dans Semen, 33, 2012, p. 121-162.

60. Par convention de transcription. / : retour à la ligne dans le corps du paragraphe.

61. É. Benveniste, Baudelaire, fo 305, p. 644.

62. Ibid., fo 311, p. 656.

63. Gérard Dessons l'avait déjà remarqué : « Il faut remarquer la valeur de l'adverbe " seulement " dont la rature montre l'hésitation entre la conception d'une double signifiance du mot (signifier et évoquer) et celle d'une signifiance unique (évoquer) qui semble être ce vers quoi tend la pensée de Benveniste : en poétique, le mot ne signifie pas (ou ne signifie plus), il évoque ", dans « Le Baudelaire de Benveniste entre stylistique et poétique », Semen, 33, 2012, p. 55-70.

64. H. Meschonnic, Poétique du traduire, cit., p. 12.

65. É. Benveniste, "Sémiologie de la langue », [1969], dans Problèmes de linguistique générale, 2, Paris, Gallimard, 1974, p. 66.

66. F. Durand-Bogaert, «Ce que la génétique dit, la traduction le fait », dans Genesis, 38, 2014, en ligne, https://genesis.revues.org/992. 


\section{RÉSUMÉS}

Les études sur l'autotraduire permettent d'enrichir la pensée du traduire dans son ensemble. En se focalisant sur le sujet (auto)traduisant, bien visible, elles rappellent l'activité, le mouvement du traduire, et non seulement le produit fini traduit. Elles replacent notamment l'attention sur l'écriture, la grande oubliée de la critique de la traduction. De ce fait, elles invitent à réfléchir sur Baudelaire d'Émile Benveniste (2011) qui nous conduit au dépassement de la dualité du signe et elles incitent à reprendre la lecture de la Poétique du traduire d'Henri Meschonnic (1999). Avec ce regard sur l'écriture du sujet (auto)traduisant, les études de la genèse des textes (auto)traduits ouvrent de nouvelles perspectives pour penser le traduire.

INDEX

Mots-clés : autotraduction, poétique, écriture, signe, traduction 